le portiQue Le Portique

le portiQue Revue de philosophie et de sciences humaines

$19 \mid 2007$

Philosophies de l'argent

\title{
Monétisation et vie sociale
}

Viviana A. Zelizer

\section{OpenEdition}

Journals

\section{Édition électronique}

URL : http://journals.openedition.org/leportique/1131

DOI : 10.4000/leportique.1131

ISSN : $1777-5280$

Éditeur

Association "Les Amis du Portique"

Édition imprimée

Date de publication : 1 janvier 2007

ISSN : 1283-8594

\section{Référence électronique}

Viviana A. Zelizer, « Monétisation et vie sociale », Le Portique [En ligne], 19 | 2007, mis en ligne le 15 décembre 2009, consulté le 25 mars 2021. URL : http://journals.openedition.org/leportique/1131 ; DOI : https://doi.org/10.4000/leportique.1131

Ce document a été généré automatiquement le 25 mars 2021.

Tous droits réservés 


\title{
Monétisation et vie sociale
}

\author{
Viviana A. Zelizer
}

La monétisation : un assèchement des relations humaines ?Quelques aspects de l'imaginaire littéraire de l'argent

1 Paris, en 1960, telle qu'elle était imaginée de manière plutôt sombre, un siècle plus tôt, par le jeune Jules Verne, est devenue une ville régulée par le pouvoir inexorable de l'argent et de la technologie : tout sentiment, tout honneur et toute imagination y est broyé par l'efficacité des structures commerciales. Dans ce Paris futuriste - tel que le rend Paris au xxe siècle, le manuscrit récemment (1994) retrouvé de Verne ${ }^{1}$ - «le besoin de devenir riche à tout prix » a fini par tuer le sentiment. Les couples amoureux, devenus des partenaires indifférents conduits par l'intérêt, ont perdu même le vocabulaire de l'affection. Les mots "pénates, dieux du logis, un intérieur, des compagnons de vie» (p. 144) ne peuvent plus être trouvés que dans des dictionnaires obsolètes. L'impérialisme de l'argent, dans ce futur contre-utopique, a remplacé tout simplement le souffle du politique et même les guerres internationales ont été neutralisées par les intérêts commerciaux : les livres anglaises, les roubles russes et les dollars américains se sont fondues en une seule monnaie de pouvoir et de domination s'exerçant sur toute la France.

2 Paris en 1996, selon le portrait qu'en trace un best-seller, fait écho de manière terrifiante à celui de Jules Verne. Dans l'Horreur économique, Viviane Forrester ${ }^{2}$, romancière à ses heures, bien qu'elle se concentre sur la disparition du travail, explique cette catastrophe comme un pur effet de l'accumulation de l'argent et de la diffusion internationale de la spéculation monétaire. La formidable économie de marché transnationale, selon Forrester, ne se soucie que de «masses monétaires et de jeux financiers » : la " démultiplication vertigineuse de la quantité de valeurs tous azimuts que /les puissances privées/ peuvent embrasser, dominer, combiner, dupliquer sans se préoccuper des lois et des contraintes qu'elles sont à même, dans un contexte ainsi mondialisé, de contourner facilement » (p. 36-37).

3 Ainsi, des observateurs français, pendant plus d'un siècle, ont insisté sur le pouvoir de standardisation et de corruption de l'argent. On trouve des arguments similaires chez des commentateurs anglo-saxons. 
Prenez la vision de l'Amérique en 2020 que donne John Updike, ce moderne Jules Verne. Dans Toward The End of Time - sélectionné par le New York Times comme l'un des meilleurs livres de $1979^{3}$ - Ben Turnbull, un conseiller en investissement retraité de 66 ans, qui est le narrateur de la peu engageante fantaisie de science-fiction d'Updike, nous raconte une Amérique post-nucléaire en ruines, broyée militairement par la Chine (Updike, 1997). Au xxie siècle, dans la ville du Massachusetts, où vit Turnbull, le "welder ", un bon au porteur couleur sépia - " ainsi nommé en l'honneur d'un célèbre gouverneur Républicain de la période d'avant-guerre» (p.14) - a remplacé, après l'effondrement de l'économie nationale, l'inutile et hyperdévalué dollar fédéral.

5 Bien que la version d'Updike tourne principalement autour de l'angoisse existentielle d'un Turnbull vieillissant, l'argent entre dans son conte avec la même capacité de corruption et d'anéantissement des sentiments que chez Verne. «Dans notre nouveau monde, on est prêt à tout pour un welder» (p. 50), souligne-t-il. En raison de l'effondrement du gouvernement fédéral, les citoyens doivent payer des gangsters pour les protéger, tandis que de jeunes prostituées, ainsi que l'explique Turnbull, négocient le sexe au moyen d'efficaces routines de marchandage. Comme le dit Turnbull, avec Deirdre, une jeune femme qui effectue des prestations à domicile, pendant l'absence de sa femme, «nos jeux amoureux sont empreints de cette excitation propre à une vente aux enchères, car elle me propose, d'une voix chaude à peine audible, de se livrer ou de se soumettre à des actes qui n'ont rien à voir avec la simple position du missionnaire » (p. 51) et cela pour un supplément de welders authentifiés avec soin.

Dans l'histoire d'Updike, la monétisation ${ }^{4}$ convertit l'intimité en une froide relation de marché. Pour le journaliste britannique James Buchan, un Âge de l'Argent - aussi déplaisant soit-il - n'est pas une fiction cauchemardesque. L'argent a déjà été « intronisé comme le Dieu /de/notre temps » (1997, p. 14). Dans son Frozen Desire : The Meaning of Money ${ }^{5}$, après un éloquent récapitulatif historique et littéraire des trajectoires sociales de l'argent, Buchan évoque la victoire finale de l'argent par laquelle "les relations des être humains, à la fois aux choses et au monde des objets " ont pris " quelques uns des traits de l'argent... fluide, temporaire, indifférent, instable » (p. 269). C'est un effet dangereux car l'argent «loin d'être l'arène indolore de l'émulation humaine, comme le soutiennent ses apologistes, est un grand destructeur... parce que l'argent est tout pouvoir et potentialité, le monde extérieur est une pauvre chose qui peut être altérée et exploitée sans vergogne»(p.278). L'éthique rend inévitablement les armes devant l'avarice : «les choix moraux sont ainsi simplement achetés; ou plutôt, comme pour la prostitution ou le cambriolage adolescents, la mémoire déformée de l'instruction morale se dissout dans les impératifs du baiser " (p. 270). En fait, Buchan fait écho aux mornes visions du futur de Verne et d'Updike :

J'imagine que le nivellement dû à l'argent, l'impatience face, à la fois, au chevaleresque et au manque de courtoisie, en viendront à dissoudre les distinctions de sexe. Le monde mélancolique de Jenny Marx en termes de Kirche, Küche, Kinder, a explosé ; et, malgré les réactions face à cette réorganisation cataclysmique de la sphère la plus intime de l'existence, comme cela s'est produit en Iran, l'argent conserve un pouvoir d'inertie. Les prétendues vertus féminines éternelles de fidélité, chasteté, piété et sens de l'épargne perdent leur féminité et cessent progressivement d'être des vertus. À l'Ouest, hommes et femmes expérimentent maladroitement leurs rôles respectifs traditionnels... Les enfants seront élevés, comme dans l'antique Sparte et dans les modernes États-Unis, par des corporations asexuées, qui imposeront à leurs employés un célibat aussi exigeant que le règlement des Shakers dans l'ancienne Nouvelle-Angleterre. Les différences entre 
les sexes s'atténueront pour se réduire au physique et peut-être même au-delà : il est déjà plus difficile de distinguer les sexes sur la Plage de Malibu que dans les rues de Kaboul, (p. 174-175).

7 Les conclusions de Buchan sont des plus surprenantes étant donné le début de son livre qui offre une interprétation très sensée de l'encastrement de l'argent dans les relations sociales, comme l'illustrent ce qu'il dit des modalités subtiles de sa rémunération en tant que jeune journaliste travaillant à Jeddah pour un journal saoudien. Ainsi, une nouvelle fois, l'argent est le grand solvant.

Verne, Forrester, Updike, Buchan : différentes intrigues, mais un seul scénario sousjacent. Tous les quatre partagent une puissante rhétorique de la monétisation, dans laquelle l'argent apporte l'efficacité, au prix d'un assèchement des relations sociales, d'une perturbation des valeurs humaines et d'une dessication des sentiments.

Impasses causalistes dans les sciences sociales

Plus généralement, les représentations populaires de la monétisation soulèvent trois questions récurrentes qui interpellent aussi les sciences sociales. Premièrement, y a-t-il des formes de relations sociales qui sont incompatibles avec l'échange monétaire? Deuxièmement, la monétisation transforme-t-elle inévitablement la qualité de la vie sociale? Et troisièmement, quelles sont les causes et les conséquences des différents types de transferts monétaires ? La fort utile évaluation du sujet que l'on doit à Andrew Leyshon et Nigel Thrift, nous permet de faire le point sur l'état de la question dans les sciences sociales actuelles. Selon Leyshon et Thrift, leur très panoramique ensemble d'essais - Money Space : Geographies of Monetary Transformation - vise à troubler les conceptions standards par une analyse de l'argent comme "processus social» (p. xiii) ${ }^{6}$.

10 Non, répondent-ils à la première question : l'argent n'est pas isolé des autres relations sociales non-économiques, parce qu'il est lui-même basé sur des "réseaux sociaux particuliers superposés qui fournissent le fondement de la signification sociale de l'argent» (p.xv). Probablement non, répondent-ils à propos des pouvoirs de modification qu'aurait l'argent, parce que ces pouvoirs ne font que contrebalancer la victoire supposée des relations marchandes, donnant «aux liens de confiance perçus un poids égal à celui des liens de confiance réels dans un grand nombre de transactions monétaires" (p.xv). Même la monnaie virtuelle «consiste en une série de pratiques sociales " (p. 21). Cependant, quand nous en venons à la question de la causalité, l'analyse est quelque peu défaillante. Leyshon et Thrift dégagent deux «discours monétaires » dominants : l'un est de suspicion (l'argent y a la force inébranlable et corrosive qu'il revêt dans l'imagination populaire); l'autre est de libération (où l'argent apporte un éclairage). Il manque une reconnaissance suffisante du fait que les deux discours partagent une histoire causale commune, où la monnaie rationalise inévitablement, en bien ou en mal.

11 Cette vision causale demeure prévalente dans toute la littérature contemporaine sur la monétisation. Considérons l'analyse très discutée que la théoricienne du droit Margaret Jane Radin ${ }^{7}$ propose du débat sur la marchandisation. Préoccupée par la «rhétorique du marché » et par sa "conception économiste de la vie»(p.6,3), Radin tente de justifier un corps de lois qui pourrait distinguer et réguler l'espace qu'elle appelle de marchandisation incomplète - où "les valeurs de la personne et de la communauté interagissent avec le marché de façon invasive et altèrent la pure forme a-marchande de beaucoup de choses » (p. 114). Pourtant, malgré son insistance sur l'interaction de la culture et de la loi, aussi bien que ses objections bien connues contre ce qu'elle appelle 
la théorie des « dominos » de la marchandisation, la position de Radin implique que la «marchandisation complète » apparaîtrait dès lors que la monétisation ne serait pas assortie de de protections institutionnelles - en particulier légales. En dépit de la grande sophistication de ses analyses à propos de l'argent et de la loi, Radin ne peut en fin de compte abandonner la théorie causale classique: l'argent livré à lui-même rationalise et corrompt.

Le renouveau des approches de l'argent dans les sciences socialesUne prolifération de travaux empiriques sur l'encastrement des rapports monétaires

Néanmoins, on peut apercevoir des signes de clarification, à mesure qu'un certain nombre de chercheurs aux perspectives disciplinaires différentes contestent le scénario causal indiscuté des effets de la monétisation. Les anthropologues ont trouvé plus d'écho que leurs collègues des autres sciences sociales en proposant une version revue de la théorie causale, qui démontre la totale interférence des transactions monétaires et de la construction des relations sociales et des systèmes de signification. C'est le cas particulièrement du livre de Jane I. Guyer, Money Matters ${ }^{8}$, recueil important d'études sur l'histoire des monnaies de l'ouest africain, qui remet en question l'idée selon laquelle les devises européennes ont chassé les monnaies "primitives» qui étaient auparavant propres aux colonies européennes d'Afrique.

la même veine, on peut souligner l'importance du volume d'essais de Jonathan Parry et Maurice Bloch, Money \& the Morality of Exchange ${ }^{9}$, qui conteste les pouvoirs d'homogénéisation universelle de la monnaie en montrant, détails ethnographiques convaincants à l'appui, la variabilité culturelle de l'argent. Plutôt qu'un " acide qui dissout inexorablement les distinctions culturelles auxquelles nous tenons tant, qui érode les différences qualitatives et réduit les relations interpersonnelles à l'impersonnalité » (p. 6), l'argent, pour Parry et Bloch est marqué par «la matrice culturelle dans laquelle il est incorporé » (p. 21); au lieu de subvertir les liens sociaux, l'argent sert souvent d' "instrument de leur maintien» (p.22). Le masque de la "mythologie économique» dominante de l'argent, nous dit Bloch dans son introduction au recueil d'essais intitulé «Les usages de l'argent ${ }^{10}$, ne tombe pas seulement parce que les sociétés non-occidentales apparaissent comme trop exotiques pour le porter : même dans les états capitalistes les plus avancés, souligne Bloch, les relations sociales significatives des gens marquent leurs transferts monétaires ${ }^{11}$.

On trouve aussi chez quelques psychologues et sociologues un début de reconnaissance de l'encastrement social et cognitif des transactions monétaires contemporaines ${ }^{12}$. En psychologie, des études récentes rejettent l'idée que l'argent correspond à un trait psychologique général et suggèrent au contraire qu'il implique «de multiples symbolisations». Toute une stimulante littérature consacrée à la «comptabilité mentale » attaque l'hypothèse des économistes quant à la fongibilité, en montrant de quelles manières les individus distinguent entre différentes sortes de monnaies. Par exemple, ils traitent une rentrée inespérée très différemment d'un dividende ou d'un héritage, même lorsque les sommes concernées sont identiques ${ }^{13}$. Des travaux proches soulignent l'impact comportemental des «illusions monétaires » par lesquelles, dans différentes situations, les gens adoptent une série de conventions locales pour évaluer les transactions monétaires plutôt que de les traduire dans le vecteur neutre et fongible des économistes ${ }^{14}$.

Le politiste Robert E. Lane ${ }^{15}$ a aussi présenté des résultats concernant une grande variété de modèles cognitifs selon lesquels les Américains pensent l'argent comme 
susceptible de variation, faisant de lui un symbole significatif d'attitudes telles que l'insuffisance personnelle, la perte de contrôle, l'échec honteux, la sécurité ou le besoin d'approbation sociale ${ }^{16}$. De la même façon, une recherche de l'anthropologue cognitiviste Jean Lave ${ }^{17}$, effectuée sur les pratiques arithmétiques quotidiennes - elle a suivi 35 hommes et femmes du Comté d'Orange, Californie, dans diverses circonstances, telles que les achats dans une épicerie et elle a examiné leurs pratiques domestiques de management monétaire - montre que ses enquêtés ne traitaient pas le revenu familial comme une "source globale de fonds familiaux (comme une somme de nature mathématique), utilisable pour de multiples buts", mais au contraire qu'ils compartimentaient leurs fonds selon différentes «cachettes" qui «reflétaient et étaient aussi porteuses des relations sociales et des catégories d'activités suivant lesquelles les gens organisent leur vie» (p. 133). L'argent, conclut Lave, « est utilisé aussi bien pour préserver les catégories morales et les relations familiales que pour les exprimer » (p. 141).

Dans Marriage Money: The Social Shaping of Money in Marriage and Banking ${ }^{18}$, la sociologue Supriya Singh va plus loin au cœur des économies domestiques, en examinant les pratiques monétaires de couples de classes moyennes australiennes, d'origine anglo-saxonne. Singh montre que les relations sociales créées par le mariage donnent forme aux transferts monétaires des épouses et des maris, tout comme à leur usage des institutions financières. Le compte commun bancaire, par exemple, est « un indicateur essentiel des manières selon lesquelles les couples construisent le sens de l'argent du mariage » (p.136), en ce qu'il affirme l'unité du lien marital: ainsi, selon Singh, peu de co-habitants partageaient un compte. Nous apprenons non seulement que les couples australiens différencient et séparent leurs monnaies selon la source et le but du transfert, mais, plus spécifiquement, comment ils utilisent différents types de stratégies bancaires (par exemple: les dépôts, les comptes d'emprunts; les comptechèques; l'épargne à court terme; les virements automatiques; les comptes à but spécifique; les comptes joints; les comptes séparés; les comptes fiduciaires; les comptes commerciaux; les comptes personnels) et différentes formes de paiement (par exemple: en liquide, par carte de crédit, par chèque, par virement, par une monnaie interne), pour marquer des devises socialement distinctes.

Les résultats de Singh nous rappellent que les gens utilisent différents lieux, à l'extérieur et à l'intérieur du logement, pour singulariser (earmark) leurs devises selon leurs relations sociales. Par exemple, des études sur l'histoire et la situation présente des économies domestiques américaines et britanniques, y compris dans le cas des logements sociaux, ont montré que différents membres de la famille, épouses, maris, enfants et quelquefois d'autres parents, peuvent entrer en lutte les uns avec les autres et avec les autorités - à propos de la définition, de l'allocation et de la régulation de leurs monnaies domestiques ${ }^{19}$.

Même dans le domaine du paiement du travail - où les explications en termes d'efficacité ont typiquement prévalu - des chercheurs ont commencé à identifier l'interdépendance des systèmes de paiement et des contextes culturels et relationnels. On songe tout particulièrement à l'analyse détaillée que Richard Biernacki ${ }^{20}$ propose des industries textiles lainières britannique et allemande : il conclut que, sur un fond semblable d'équipement économique et technique, les différences de pratiques dans les usines britanniques et allemandes, y compris les modes de rémunération, sont dues à des différences nationales dans la façon de définir le travail comme une marchandise. 
L'ethnographie très acérée que David Stark ${ }^{21}$ nous a donnée d'une usine socialiste hongroise durant les années 1984-1986, nous plonge dans un monde de paiement délibérément différencié au sein d'un même contexte culturel. Après s'être organisé en partenariat contractualisé pour produire des biens et les vendre durant le temps horstravail - utilisant pour cela les locaux et les technologies de leur activité salariée - un groupe de travailleurs qualifiés avait aussi inventé un système de paiement pour marquer le caractère séparé de cette identité collective indépendante. Leur paie individuelle normale, définie par le management, comprenait trois composantes: le salaire normal, les heures supplémentaires et des "salaires variables" comme les bonus. À la place du système normal, les partenaires négocièrent via leurs représentants élus, un ensemble de "rétributions d'entrepreneurs ", qui fut ensuite réparti entre les travailleurs participants. Bien que ce processus ait entraîné des contestations au sein du groupe et parmi les sous-contractants, managers et autres travailleurs, l'analyse de Stark montre bien la création de systèmes de paiement représentant différents aspects de relations sociales ${ }^{22}$. Il y a deux manières, l'une cognitive et l'autre relationnelle, d'interpréter ce qui est en jeu dans ces pratiques de marquage monétaire : soient, deux processus, distincts quoique interdépendants. Selon la première, les variations montaires sont des distinctions mentales ou des cartes cognitives; selon l'autre, la différenciation monétaire émerge des relations sociales différenciées et des systèmes de significations partagées et elle est marquée par eux. Ainsi, les chercheurs en sciences sociales ne se contentent pas de nous rapporter des stratégies budgétaires individuelles sophistiquées, mais ils identifient la signification des pratiques monétaires comme tout à la fois le résultat, le contenu et l'origine des relations sociales. Ces deux processus sont évidemment à l'œuvre, mais la recherche actuelle se limite à examiner la relation donner-prendre dans la création, le maintien et la transformation des catégories de paiement ${ }^{23}$.

À l'horizon : la question des liens sociaux

19 Plus généralement, l'objectif de cette recherche trans-disciplinaire pionnière sur l'argent n'est pas de démontrer comment quelques domaines des relations sociales tiennent bon face aux effets de la marchandisation. La critique va bien au-delà d'une identification d'épisodes exceptionnels de résistance à un marché dominant; elle soutient que les gens utilisent de façon routinière les transactions monétaires - comme ils le font pour d'autres médias culturels - pour créer, définir, affirmer, se représenter, contester ou renverser leurs liens sociaux. Non pas que les analyses les plus récentes de l'argent l'aient traité comme un bien pur : il ne s'agit pas de nier que les gens puissent faire le mal par des transactions monétaires. Ce qui découle de cette analyse c'est que les gens peuvent construire et effectivement construisent des relations impliquant l'argent et qui sont étriquées, passagères, hostiles ou altérées, tout comme cela peut donner lieu à des liens sociaux riches, durables et affectueux. Les processus conduisant à ces deux issues, impliquent l'un comme l'autre, la construction de relations sociales pleines de sens, simplement dans le premier cas, elles sont construites pour être étroites et passagères et, dans le second, pour être durables et larges.

Récemment, mon propre livre sur le sujet a proposé un suivi de l'intersection des paiements monétaires et de différentes sortes de liens sociaux. Après avoir rendu compte de la standardisation de l'offre légale au xixe siècle aux États-Unis, ce livre, Social Meaning of Money ${ }^{24}$, a examiné les changements des pratiques monétaires américaines sous l'angle des économies du don, de l'aide sociale et des économies domestiques depuis la fin du xixe siècle. Prenant à contre-pied l'attente commune 
d'une monétisation de ces pratiques qui conduirait à restreindre et à dessécher les relations sociales concernées, il montrait des Américains déployant une grande détermination et ingéniosité pour refaçonner les transactions monétaires en rapport avec le changement des liens sociaux. Depuis la fin de ce livre, j'ai surtout observé les pratiques monétaires dans deux directions: la création de systèmes de paiement différentiels à l'intérieur des entreprises américaines; et l'inscription de transferts monétaires dans les relations intimes, impliquant la parenté, le soin domestique et /ou la sexualité. Ce dernier sujet m'a conduite à analyser des procès, puisque les tribunaux américains ont été, de façon tout à fait typique, peu enclins à reconnaître la compatibilité des transferts monétaires et de l'intimité, mais que les questions de juste compensation ont souvent soulevé des problèmes de ce type. Une fois encore, mes investigations ont révélé un grand effort et une grande ingénuité des humains lorsqu'il s'agit de concilier les paiements monétaires et les liens sociaux ${ }^{25}$.

De nouvelles avancées théoriques sur l'encastrement des relations économiques

21 La modification de notre compréhension de l'argent et des paiements relève d'une réinterrogation plus générale des processus économiques. Comme l'illustre le très marquant Handbook of Economic Sociology ${ }^{26}$, un très grand éventail de chercheurs qu'il s'agisse de psychologues, de chercheurs en sciences sociales, de juristes et de spécialistes de littérature - prennent à présent au sérieux l'interaction entre le contexte social et les transactions économiques ${ }^{27}$. Renversant une tendance qui permettait de plus en plus souvent aux analyses économiques de fournir l'explication des phénomènes sociaux, cette recherche trans-disciplinaire utilise fréquemment des concepts et des outils sociologiques pour interpréter des transactions économiques de grande ampleur.

Voyez, par exemple, ce qu'on appelle la « Nouvelle École de Chicago » en droit : entrant en lutte contre l'hégémonie historique du paradigme "droit et économie », un groupe d'analystes du droit redécouvre le pouvoir explicatif et le potentiel, en termes de politiques, des normes et des significations sociales ${ }^{28}$. De plus en plus, les investigations des chercheurs en droit les conduisent à des confrontations ou à des collaborations avec des chercheurs en sciences sociales. Ces derniers insistent en général moins sur la fabrication des lois effectives que sur l'explication des pratiques qui posent problème au droit. Néanmoins, des deux côtés, on voit émerger une interprétation plus complète des interférences de la monétisation et de la vie sociale.

Des travaux récents sur l'argent ont fait apparaître une question encore plus fascinante pour la réflexion et la recherche : pourquoi et comment ces différentes rhétoriques de l'argent - dénonciatrices ou élogieuses - ont-elles autant d'attrait? Ainsi, Ira Katznelson ${ }^{29}$ s'est récemment demandée pourquoi ces conceptions sont-elles «si séduisantes et si influentes en tant que représentations de la réalité sociale? Plus précisément, pourquoi des secteurs aussi distincts que l'État, le marché et la société civile semblent-ils si assujettis à aussi peu ?» (p. 97). Katznelson attire l'attention sur la division historique entre souveraineté et propriété en Occident, qui se ramifie en séparations au sein de l'Etat, de l'économie et de la société civile. Pour Katznelson, l'attrait de la rhétorique de la marchandisation repose sur des interprétations plus fondamentales concernant la nature de l'organisation sociale.

Robert Wuthnow apporte une réponse différente dans son ouvrage Poor Richard's Principle ${ }^{30}$ : après interview de 200 répondants et une analyse d'enquêtes sur foi, travail et argent, Wuthnow établit un lien entre l'attrait de ces rhétoriques et 
l'accroissement de la séparation culturelle et institutionnelle de la vie privée et de la vie publique aux États-Unis. Comme l'argent, dans l'arène institutionnelle publique est lié à la rationalité, à l'objectivité et à l'efficacité, dans la sphère privée, l'argent est de plus en plus subjectivisé, tiré du côté d'une profonde anxiété personnelle, d'une responsabilité individuelle et presque d'un sujet tabou ne souffrant aucune infraction et ne pouvant être abordé avec d'autres qu'en des circonstances exceptionnelles. Au regard de ce que dit Katznelson, Wuthnow met davantage l'accent sur l'ancrage des idées concernant la marchandisation dans l'altération actuelle des institutions sociales fondamentales.

L'œuvre de Jeffrey Alexander ${ }^{31}$ (1995) offre néanmoins une troisième explication possible de l'attraction exercée par la rhétorique de la marchandisation. À la différence de Katznelson (op. cit., 1997) et de Wuthnow (op. cit., 1996), Alexander trouve fascinante la réapparition d'évaluations positives de la marchandisation. Bien sûr, les critiques du capitalisme, depuis Marx, ont trouvé séduisante l'opinion selon laquelle la marchandisation, en tant que porteuse emblématique d'un capitalisme oppressif, à la fois rationalise et comprime nos liens sociaux. Alexander fait toutefois remarquer qu'à la fin des années quatre-vingt-dix, la rhétorique anti-capitaliste est concurrencée par le renouveau des marchés à la fois dans les économies capitalistes et post-socialistes. L'évaluation d'Alexander, dans son Fin de Siècle, conduit à identifier une "théorie sociale positive des marchés » de type néo-moderne. Répondant au leg du socialisme, "différents groupements d'intellectuels contemporains ont - selon Alexander regonflé le récit émancipateur du marché », rendant la libération «dépendante de la privatisation, des contrats, des inégalités d'argent et de la compétition» (p. 32). Ainsi, Alexander traite le changement institutionnel et la transformation intellectuelle comme des résultats d'événements historiques majeurs; la conséquence, suggère-t-il en est une nouvelle crédibilité pour des évaluations positives de la marchandisation.

Ainsi, le débat avance. Nouveau point à l'ordre du jour : l'explication de l'attractivité de différentes rhétoriques concernant la marchandisation. En procédant ainsi, nous pouvons retourner avec profit aux distinctions figurant dans la théorie rivale de la société de marché que présentait A. O. Hirschman ${ }^{32}$. Bien entendu, l'analyse issue des sciences sociales quant aux processus économiques a déjà sapé les fondements des distinctions tranchées entre des sphères monétaires et non-monétaires dans la vie sociale contemporaine. On n'a pas pourtant pas tenu compte de ce que Katznelson (op. cit.) identifie comme l'attrait rhétorique persistant des idéologies de la marchandisation. Alexander (op. cit.) rattache la revitalisation de la sociologie économique à la même série de changements qui à présent légitime les rhétoriques du marché. Selon Alexander, l'intérêt renouvelé pour l'encastrement culturel et structurel de l'action économique débouche sur une image du marché comme "une relation sociale et interactionnelle qui ressemble peu à ce qui, dans le passé, était vu comme l'exploitation capitaliste sans attaches » (p. 33).

Quelle que soit la solution de cette importante question d'histoire intellectuelle soulevée par Alexander, ce dernier attire à juste titre l'attention sur ce que peut avoir de stimulante la prise en considération de l'ingéniosité qui est maintenant engagée dans les interactions entre les transactions marchandes et d'autres aspects de la vie sociale et culturelle.

Paru en anglais sous le titre : "Monetization and social life », dans Etnofoor, 13 (2000), p. 5 à 15. Que la revue Etnofoor soit chaleureusement remerciée pour l'aimable 
autorisation donnée à cette traduction (NDT). Cet article a été traduit par Jean-Yves Trépos. Les intertitres ont été élaborés par J.-Y. Trépos, avec l'accord de V. Zelizer, spécialement pour cette traduction.

\section{NOTES}

1.. Jules VERnE, Paris au $X^{e}$ siècle, Paris, Hachette, 1994.

2. Viviane FORRESTER, L'Horreur économique, Paris, Fayard (cité dans l'édition du Livre de Poche), 1996.

3.. John UPDIKE, Toward The End Of Time, New York, Alfred A. Knopf, 1997 (Aux confins du temps, traduit par C. et J. Demanuelli, Paris, Seuil, 2000).

4.. Le choix de traduire « monetization » par " monétisation » et non par « monétarisation » a été fait en accord avec Viviana Zelizer, par souci d'éviter toute confusion avec les thèses de l'école monétariste (N.D.T.).

5.. James BUCHAN, Frozen Desire : The Meaning of Money, New York, Farrar Straus Giroux, 1997.

6.. Andrew LEYSHON \& Nigel THRIFT, Money Space : Geographies of Monetary Transformation, London, Routledge, 1997.

7.. Margaret Jane RADIN, Contested Commodities, Cambridge, Harvard University Press, 1996.

8.. Jane I. GUYER (ed.), Money Matters, Portsmouth (NH), Heinemann, 1995.

9.. Jonathan PARRY, \& Maurice BLOCH (eds), Money \& The Morality of Exchange, New York, Cambridge University Press, 1989.

10.. Maurice BLOCH, «Les usages de l'argent », Terrain 23, 1994, p. 5-10 (« Les usages de l'argent ", sous la responsabilité de $\mathrm{M}$. Bloch).

11.. Voir aussi David AKIN, \& Joel RoBBINS (eds), Money and Modernity: State And Local Currencies In Melanesia, Pittsburgh (Pa.), University of Pittsburgh Press, 1999 et Heinzpeter ZNOJ, « Hot Money and War Debts : Transactional Regimes in Southwestern Sumatra ", Comparative Studies in Society and History 40 (April), 1997, p. 193-222. 12.. Soient quelques exemples empruntés à différentes disciplines, de travaux sur l'argent : Lapo BERTI et al., «Il Denaro », Problemi del Socialismo 7-8, 1991 ; Nigel DODD, The Sociology of Money, New York, Continuum, 1994 ; Kenneth O. DOYLE (ed), « The Meanings of Money ", American Behavioral Scientist 35, 1992 ; Jane I. GUYER (ed.), Money Matters, Portsmouth (NH), Heinemann, 1995 ; Emily GILBERT \& Eric HELLEINER (eds.), Nation-States and Money: The Past, Present and Future of National Currencies, London, Routledge, 1999 ; Keith HART, The Memory bank : Money in an Unequal World, London, Profile Books, 1997 ; M. S. MIZRUCHI \& L. Brewster STEARNS, « Money, Banking, and Financial Markets », in Neil SMELSER \& Richard SWEDBERG (eds.), The Handbook of Economic Sociology, Princeton (N.J.), Princeton University Press and New York, Russell Sage Foundation, 1994, p. 313-341 ; Carlo MONGARDini (ed.), Il denaro nella cultura moderna, Roma, Bulzoni, 1998 ; Marc SHELL, Art \& Money, Chicago, University of Chicago Press, 1995 ; David woodRUFF, Money Unmade : Barter and the Fate of Russian Capitalism, Ithaca (N.Y.), Cornell University Press, 
1999 ; Robert wUTHNow, God and Mammon in America, New York, Free Press, 1994 ; Robert wUTHNow, Poor Richard's Principle, Princeton (N.J.), Princeton University Press, 1996 ; Geoffrey INGHAM, " On the Underdevelopment of the "Sociology of Money" ", Acta Sociologica 41, 1997, p. 3-18. Pour d'autres références, voir Viviana A. ZELIZER, The Social Meaning of Money, New York, Basic Books, 1994 (La Signification sociale de l'argent, traduit par Christian Cler, Paris, Seuil, 2005).

13.. Voir, par exemple, Chip HEATH \& Jack B. solL, « Mental Budgeting and Consumer Decisions ", Journal of Consumer Research, 23, 1996, p. 40-52 ; Daniel KAHNEMAN \& Amos TVERSKY, «The Psychology of Preferences », Scientific American 246, 1982, p. 160-173 ; Richard H. THALER, Quasi Rational Economics, New York, Russell Sage Foundation, 1991 ; Richard H. THALER, The Winner's Curse, Princeton, Princeton University Press, 1992 ; Richard H. THALER, « Mental Accounting Matters ", Journal of Behavioral Decision Making 12, 1999, p. 183-206.

14.. Voir Eldar SHAFIR, Peter DIAMOND \& Amos TVERSKI, « Money Illusion », The Quarterly Journal of Economics CXII/2, 1997, p. 341-74.

15.. Robert LANE, The Market Experience, New York, Cambridge University Press, 1991.

16.. Robert LANE, The Loss of Happiness in Market Democracies, New Haven, Yale University Press, 2000.

17.. Jean LAVE, Cognition In Practice, New York, Cambridge University Press, 1988.

18. Supriya SINGH, Marriage Money: The Social Shaping of Money in Marriage \& Banking, St. Leonards, Australia, Allen \& Unwin, 1995.

19.. Voir, par exemple, Kathryn EDIN \& Laura LEIN, Making Ends Meet : How Single Mothers Survive Welfare and Low-Wage Work, New York, Russell Sage Foundation, 1997 ; Carl Husemoller Nightingale, On The Edge, New York, Basic Books, 1993 ; Shelly LUNDBERG \& Robert A. POLLAK, «Bargaining and Distribution in Marriage ", Journal of Economic Perspectives 10, 1996, p. 139-158 ; Jan PAHL, Invisible Money : Family Finances in the Electronic Economy, Bristol, Policy Press, 1999 ; Susan PORTER BENSON, « Living on the Margin : Working-Class Marriages and Family Survival Strategies in the United States, 1919-1941 ", in Victoria de GRAZIA, with Ellen FURLOUGH (ed.), The Sex of Things, Berkeley, University of California Press, 1996 ; Ellen Ross, Love \& Toil : Motherhood in Outcast London 1870-1918, New York, Oxford University Press, 1993 ; Pepper SCHWARTZ, Peer Marriage, New York, Free Press, 1994 ; Reva B. SIEGEL, « The Modernization of Marital Status Law : Adjudicating Wives' Rights to Earnings, 1860-1930 », Georgetown Law Journal, 82, 1994, p. 2127 ; Reva B. SIEGEL, « Home as Work : The First Woman's Rights Claims Concerning Wives' Household Labor, 1850-1880», Yale Law Journal, 103, 1994b, p. 1073. Pour d'autres références, voir ZELIZER, op. cit., 1994.

20.. Richard BIERNACKI, The Fabrication of Labor, Berkeley, University of California Press, 1995.

21.. David STARK, « Work, Worth, and Justice In A Socialist Mixed Economy », Working Papers on Central and Eastern Europe, Center for European Studies, Harvard University, $n^{\circ}$ 5, 1990 («La valeur du travail et sa rétribution en Hongrie », Actes de la recherche en sciences sociales $\mathrm{n}^{\circ} 85$, p. 3-19).

22.. Sur la compensation pour le travail, voir aussi le chapitre 10 in Chris \& Charles TILLY, Work Under Capitalism, Boulder (Colorado), Westview, 1998.

23.. Pour une synthèse des approches culturelle, cognitive et relationnelle des processus sociaux, voir Paul DIMAGGIO, «Culture and Cognition », Annual Review of Sociology 23, 1997, p. 263-87. 
24.. V. A. ZELIZER, op. cit., 1994.

25.. Voir, par exemple, Viviana A. ZELIZER, « Payments and Social Ties », Sociological Forum 11, (September) 1996, p. 481-495 ; Viviana A. ZELIZER, « Multiple Markets, Multiple Cultures », in Neil SMELSER \& Jeffrey ALEXANDER (eds.), Diversity and Its Discontents : Cultural Conflict and Common Ground in Contemporary American Society, Princeton (New Jersey), Princeton University Press, 1999, p. 193-212 ; Viviana A. ZELIZER, The Purchase of Intimacy, Princeton (N.J.), Princeton University Press, 2005.

26.. Neil SMELSER \& Richard SWEDBERG (eds.), The Handbook of Economic Sociology, Princeton (N. J.), Princeton University Press and New York, Russell Sage Foundation, 1994.

27. Voir Bernard BARBER, « All Economies Are "Embedded" : The Career of a Concept, and Beyond ", Social Research 62, 1995, p. 387-413 ; Avner BEN-NER, \& Louis PUTTERMAN (eds), 1998, Economics, Values, and Organization, New York, Cambridge University Press ; Michel CALLon (ed.), The Law of Markets, Oxford, Blackwell (The Sociological Review Monographs), 1998 ; Pierre BOURDIEU, « Le champ économique ", Actes de la recherche en sciences sociales $n^{\circ} 119,1997$, p. 48-66 ; Pierre BOURDIEU, Les Structures sociales de l'économie, Paris, Seuil, 2000 ; Nancy FOLBRE et al., « Debating Markets », Feminist Economics, 2, 1996, p. 69-85 ; Jean-Jacques GISLAIN \& Philippe STEINER, La Sociologie économique 1890-1920, Paris, PUF, 1995 ; Philippe STEINER, La Sociologie économique, Paris, Éditions La Découverte, 1999 ; John LIE, « Sociology of Markets », Annual Review of Sociology 23, 1997, p. 341-360 ; Shira B. LEWIN, « Economics and Psychology : Lessons For Our Own Day From the Early Twentieth Century », Journal of Economic Literature XXXIV, 1995, p. 1293-1323 ; Michael PIORE, "Review of The Handbook of Economic Sociology ", Journal of Economic Literature XXXIV, 1996, p. 741-754 ; Alejandro PORTES (ed.), The Economic Sociology of Immigration, New York, Russell Sage Foundation, 1995 ; Richard SWEDBERG (ed.), Explorations in Economic Sociology, New York, Russell Sage Foundation, 1993.

28. Voir, par exemple, Lawrence LESSIG, « The Regulation of Social Meaning ", The University of Chicago Law Review 62, 1995, p. 943-1045 ; Lawrence LESSIG, " Social Meaning and Social Norms ", University of Pennsylvania Law Review 144, 1995, p. 2181-2189; Lawrence LESSIG, « The New Chicago School », The Journal of Legal Studies XXVII (Pt. 2), 1998, p. 661-691 ; Cass R. SUNSTEIN, «Incommensurability and Valuation In Law », Michigan Law Review 92, (February) 1994, p. 779-861 ; Cass R. SUNSTEIN, Free Markets and Social Justice, New York, Oxford University Press, 1997 ; pour une tentative, voisine, de conciliation des approches économique et sociologique dans le traitement des problèmes juridiques, voir Marc GALANTER \& Mark Alan EDWARDS (ed.), « Law and society \& Law and Economics : Common Ground, Irreconcilable Differences, New Directions ", Wisconsin Law Review, 3 (Special issue), 1997.

29.. Ira KATZNELSON, « Sociology and History : Terms of Endearment? », in Kai ERIKSON (ed.), Sociological Visions, Lanham (Maryland), Rowman \& Littlefield, 1997, p. 95-99. 30.. Robert WUTHNOW, Poor Richard's Principle, Princeton (N. J.), Princeton University Press, 1996.

31.. Jeffrey C. ALEXANDER, Fin de Siècle Social Theory, London, Verso, 1995.

32.. Albert O. HIRSCHMAN, The Passions and the Interests, Princeton (N. J.), Princeton University Press, 1977 (Les Passions et les Intérêts, trad. par Pierre Andler, Paris, PUF, 1980); Albert O. HIRSCHMAN, Rival Views of Market Society, New York, Viking, 1986. 


\section{AUTEUR}

VIVIANA A. ZELIZER

Viviana A. Zelizer est professeur de sociologie à l'université de Princeton (É.U.).

Spécialiste de sociologie économique de renommée internationale, elle a surtout ouvert de nouvelles voies de recherche en sociologie de l'argent. Elle a publié : Morals and Markets: The Development of Life Insurance in the United States, New York, Columbia University Press, 1979; Pricing the Priceless Child: The Changing Social Value of Children, New York, Basic Books, 1985 (nouvelle édition revue: Princeton University Press, 1994); The Social Meaning of Money, New York: Basic Books, 1994 (La Signification sociale de l'argent, traduit en français par Christian Cler, Paris, Seuil, 2005); The Purchase of Intimacy, Princeton, N. J., Princeton University Press, 2005. 